\title{
Segmental maternal heterodisomy of the proximal part of chromosome 15 in an infant with Prader-Willi syndrome
}

\author{
Sergey Nazarenko ${ }^{1}$, Elena Sazhenova ${ }^{1}$, Alessandra Baumer ${ }^{* 2}$ and Albert Schinzel ${ }^{2}$ \\ ${ }^{1}$ Institute of Medical Genetics, Russian Academy of Medical Sciences, Tomsk, Russia; ${ }^{2}$ Institute of Medical Genetics, \\ University of Zurich, Schorenstrasse 16, CH-Schwerzenbach, Switzerland
}

Uniparental disomy (UPD) 15, detected in patients with Prader-Willi (PWS) and Angelman syndromes, has to date always involved the entire chromosome 15 . We report the first case of segmental maternal uniparental heterodisomy confined to a proximal part of chromosome 15 in a child with clinical features of PWS. This unusual finding can be explained by the rare combination of three consecutive events: a trisomy 15 zygote caused by a maternal meiosis I error, early postzygotic mitotic recombination between maternal and paternal chromatids, and, finally, trisomy rescue by the loss of the rearranged chromosome 15 containing the paternal $15 q 11-q 13$ segment.

European Journal of Human Genetics (2004) 12, 411-414. doi:10.1038/sj.ejhg.5201168

Published online 3 March 2004

Keywords: maternal segmental uniparental disomy; heterodisomy; chromosome 15; imprinting; Prader-Willi syndrome

\section{Introduction}

Among more than 100 cases of UPD for different chromosomes reported in the literature to date, the overwhelming majority shows UPD for an entire chromosome. However, UPD of segments of chromosomes with the exception of mosaicism for paternal UPD of the chromosomal segment 11p15-pter in patients with Beckwith-Wiedemann syndrome (BWS) have only occasionally been reported. ${ }^{1}$ Although maternal UPD(15) is not an infrequent finding, occurring in approximately $30 \%$ of patients with PraderWilli syndrome (PWS), and paternal UPD(15) is found in about $5 \%$ of cases with Angelman syndrome (AS), no case with UPD of only a segment including the critical region $15 \mathrm{q} 11.2-\mathrm{q} 12$ has so far been reported. This prompts us to describe the observation of segmental heterodisomic maternal UPD15 in a patient with PWS.

*Correspondence: Dr A Baumer, Universitat Zürich, Institut Für Medizinische Genetik, Schorenstrasse 16, CH-8603 Schwerzenbach, Switzerland. Tel: +41165573 25;

Fax: + 41165572 20; E-mail: baumer@medgen.unizh.ch

Received 14 October 2003; revised 15 December 2003; accepted 7 January 2004

\section{Case report}

The propositus, a male (Figure 1), was the first-born child of healthy, non-consanguineous parents. At the time of delivery, the mother was 22 years and the father 24 years old. The mother reported diminished fetal activity. The patient was born after a term uncomplicated pregnancy and induced delivery at 40 weeks. Birth weight was $3200 \mathrm{~g}$ (25th centile), and length was $53 \mathrm{~cm}$ (90th centile). His father's height is $176 \mathrm{~cm}$ and his mother's is $180 \mathrm{~cm}$. The infant had severe hypotonia and poor suck in the neonatal period. After the age of 6 months feeding difficulties improved, and he developed progressive obesity: at 9 months of age his weight was $10.0 \mathrm{~kg}$ (75th centile), and at 1 year $-12.4 \mathrm{~kg}$ (95th centile). He was referred to the Tomsk Institute of Medical Genetics at the age of 2 years for genetic evaluation with a preliminary diagnosis of PWS.

At examination at 2 years of age, the proband had severe obesity resulting from hyperphagia, convergent strabismus, full cheeks, microstomia with carp-shaped mouth, short neck, small hands with delicate and tapering fingers, and small feet (foot length $11.6 \mathrm{~cm},<3$ rd percentile). He also had cryptorchidism with hypoplastic penis 


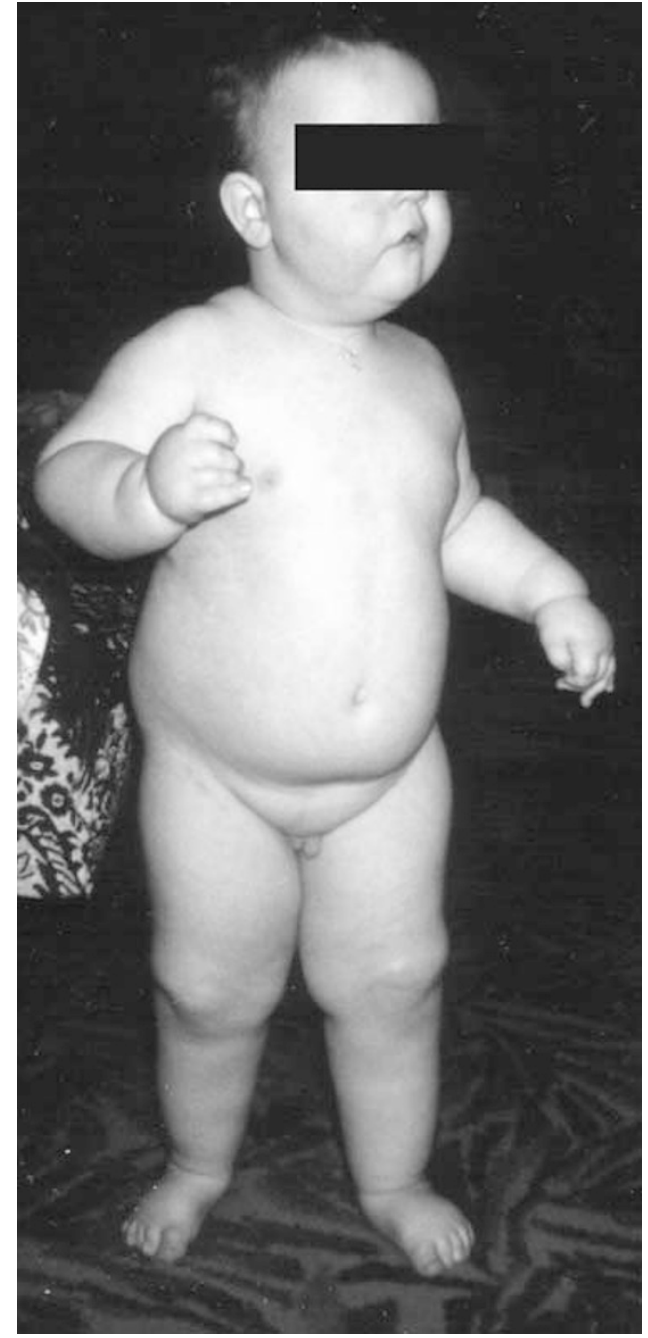

Figure 1 Proband $\mathrm{E}$ at age 2 years old.

and scrotum, muscular hypotonia, hyporeflexia, retarded psychomotor, and speech development. His weight was $20.7 \mathrm{~kg}$ (far above the 97th centile), and his length was $87 \mathrm{~cm}$ (50th centile). The phenotype is consistent with the diagnosis of PWS.

\section{Methods}

Peripheral blood samples were collected from the patient and both of his parents for cytogenetic and molecular analysis. Peripheral blood lymphocytes were cultured according to standard techniques, followed by staining with GTG and DA-DAPI. Fluorescence in situ hybridization (FISH) was performed according to the manufacturers' protocols using the commercially obtained locus-specific DNA probes SNRPN/PML and D15S10/PML (Appligene and Oncor).
The analysis of methylation status of the SNRPN gene, in the PWS critical region 15q11-q13, was done by methylation-specific PCR using DNA treated with sodium bisulfite. $^{2}$ Genomic DNA was purified using a commercial kit (Promega, Madison, WI, USA) and microsatellite analysis was performed according to standard protocols.

\section{Results and discussion}

Conventional chromosome analysis following GTG banding showed a normal male karyotype with 46 chromosomes in all 50 cells examined. The FISH results with the two DNA probes SNRPN/PML and D15S10/PML confirmed the lack of a deletion at 15q11-q13. A single band representing methylated SNRPN alleles, that is, the maternal alleles in the normal situation, was observed by methylation-specific PCR technique with DNA treated with sodium bisulfite (data not shown). This pattern is typically detected in PWS patients. The analysis of 19 polymorphic microsatellite markers distributed along

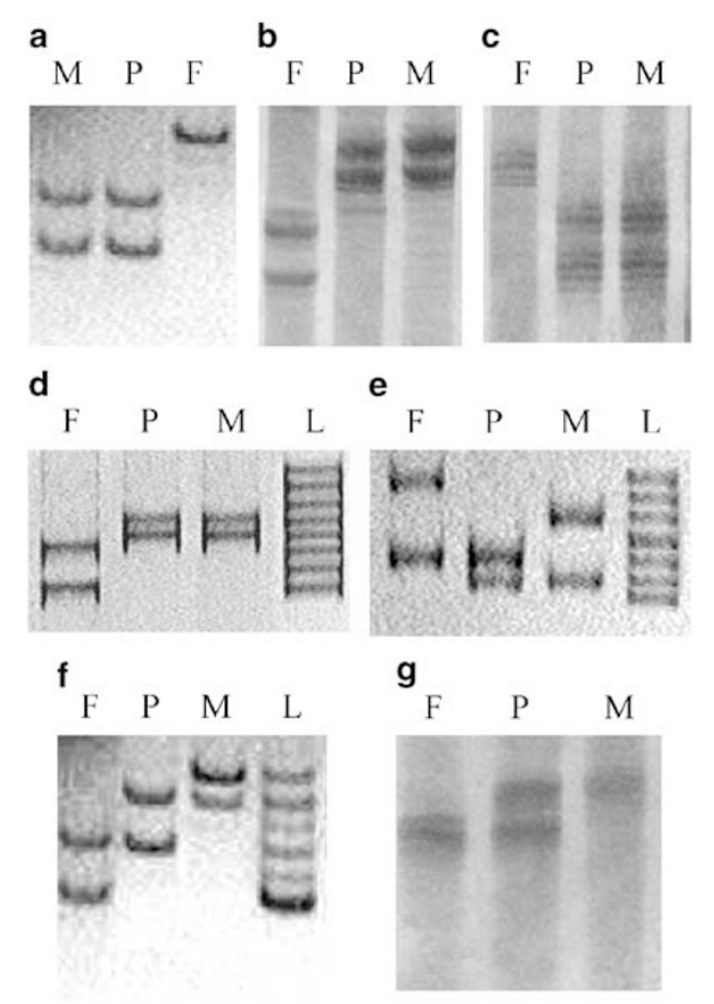

Figure 2 Microsatellite markers indicating matUPD15 at the proximal and biparental inheritance at the distal part of the long arm of chromosome 15. (a-d) matUPD15 of the $15 q 11-q 13$ region revealed by markers $D 155817$ (a), $D 1551234$ (b), D15S128 (c), and D15S172 (d). (e-g) Biparental inheritance at markers D15S643 (e), D155642 (f), and D155123 (g). F - father; P - proband; M - mother; $\mathrm{L}$ - ladder. 
Table 1 Results of chromosome 15 STR typing for our proband and his parents

\begin{tabular}{|c|c|c|c|c|c|c|}
\hline STR & Coordinate $(\mathrm{Mb})$ & Location & Father & Proband & Mother & Informativity \\
\hline D15S172 (M37) & 21.9 & q12 & $1-2$ & $3-4$ & $3-4$ & Maternal UPD \\
\hline D15S817 & 22.1 & q11.1 & $3-3$ & $1-2$ & $1-2$ & Maternal UPD \\
\hline D15S128 & 22.4 & q11.2 & $3-3$ & $1-2$ & $1-2$ & Maternal UPD \\
\hline D15S122 & 23.2 & q12.2 & $2-2$ & $1-1$ & $1-1$ & Maternal UPD \\
\hline D15S113 & 23.6 & q12 & $1-2$ & $1-1$ & $1-1$ & Uninformative \\
\hline D15S1234 & & q11.2 & $1-2$ & $3-4$ & $3-4$ & Maternal UPD \\
\hline GABRB3 & 24.2 & q12.2 & $2-4$ & $1-3$ & $1-3$ & Maternal UPD \\
\hline D15S97 & 24.4 & q12.2 & $1-2$ & $1-2$ & $1-2$ & Uninformative \\
\hline D15S217 & 25.4 & q13 & $1-1$ & $1-1$ & $1-1$ & Uninformative \\
\hline D15S1048 & 27.4 & q13.3 & $1-2$ & $1-2$ & $2-2$ & No matUPD \\
\hline D15S144 & 31.2 & q14 & $1-2$ & $1-2$ & $1-3$ & No matUPD \\
\hline D15S118 & 33.8 & q14 & $1-3$ & $1-2$ & $2-2$ & Biparental \\
\hline D15S659 & 43.8 & q15.1 & $1-2$ & $2-2$ & $2-2$ & Uninformative \\
\hline D15S123 & 45.5 & q15.2 & $1-1$ & $1-2$ & $2-2$ & Biparental \\
\hline D15S643 & 57.2 & $\mathrm{q} 22.2$ & $2-4$ & $1-2$ & $1-3$ & Biparental \\
\hline D15S98 & 57.9 & $\mathrm{q} 22.2$ & $2-2$ & $1-2$ & $1-2$ & Uninformative \\
\hline D15S100 & 93.2 & q26.2 & $1-1$ & $1-2$ & $2-3$ & Biparental \\
\hline D15S120 & 97.1 & q26.3 & $2-3$ & $2-2$ & $1-2$ & Uninformative \\
\hline D15S642 & 99.8 & q26.3 & $1-2$ & $2-3$ & $3-4$ & Biparental \\
\hline
\end{tabular}

chromosome 15 showed five fully informative markers mapping to 15q11-12.2 with two different maternal alleles and no paternal allele in the patient, indicating maternal uniparental heterodisomy of chromosome 15 (Figure 2, Table 1). However, five more distally located informative markers demonstrated biparental inheritance (Figure 2, Table 1). A maternal uniparental disomy could be excluded for the partially informative markers D15S1048 and D15S144. Thus, the uniparental disomy appears to extend to a position between $24.2(15 q 12.2)$ and $27.4 \mathrm{Mb}$ (15q13.3). Paternity was confirmed with high probability (at least $99.9 \%$ ) by the results of the analysis of seven microsatellite markers mapping to other chromosomes (data not shown).

To the best of our knowledge, only 13 cases of segmental UPD have been reported to date. These consisted of: one case with unclear parental origin of isodisomy for chromosome $4 \mathrm{p}^{3}$ eight cases with segmental maternal UPD (for chromosomes $2,4,7,14,17$, and X), ${ }^{4-11}$ and four cases with segmental paternal UPD (for chromosomes 6, 14, and 20). ${ }^{12-15}$ Cases of segmental heterodisomy are rarer than cases with isodisomy: only three cases of heterodisomy have been detected (for chromosomes 14 and 17) ${ }^{8-10}$ and all of them were maternal in origin. However, at a recent reevaluation of one case previously reported as having segmental matUPD $14,{ }^{8}$ the results of comprehensive microsatellite marker analysis did not confirm maternal UPD. ${ }^{16}$

Maternal heterodisomy 15 in our proband with PWS was observed at 15q11.1-12.2 (the most distal informative marker was GABRB3), while analysis of markers mapping to more distal segments of the long arm of chromosome 15 demonstrated biparental inheritance. The relative proneness to recombinations of the $15 \mathrm{q} 11-\mathrm{q} 13$ region may be attributed to low copy repeats derived from large genomic duplications in the vicinity of the common breakpoints. ${ }^{17}$
The mechanism of formation of segmental maternal UPD in our case appears to be the combination of three consecutive events: trisomy 15 in the zygote following a maternal meiosis I error, early postzygotic mitotic recombination between maternal and paternal chromatids, and, finally, trisomy rescue by loss of the rearranged homologue containing the paternal $15 \mathrm{q} 11-\mathrm{q} 13$ segment. Although a similar case has not, to the best of our knowledge, been reported to date, it has to be emphasized that such an event could easily be overlooked if the analysis, aimed at detecting a maternal UPD of the critical 15q11-q13 region, is not extended to more distal regions. Although it is highly advisable to test distal markers, informative markers at 15q11-13 that show heterodisomy would be per se sufficient for a molecular diagnosis (if the methylation test is also abnormal and paternity is confirmed).

Finally, the observation that our proband's phenotype was in no way different from patients with maternal UPD for the entire chromosome 15 confirms the assumption that maternal UPD of the segments beyond 15q11-q13 has no additional influence on the phenotype.

\section{Acknowledgements}

This study was supported by the Swiss National Fund (Grant SNF 7IP65679)

\section{References}

1 Kotzot D: Complex and segmental uniparental disomy (UPD): review and lessons from rare chromosomal complements. J Med Genet 2001; 38: 497-507.

2 Kubota T, Das S, Christian SL, Baylin SB, Herman JG, Ledbetter DH: Methylation specific PCR simplifies imprinting analysis. Nat Genet 1997; 16: 16-17.

3 Collier DA, Barrett T, Curtis D, Macleod A, Bundey S: DIDMOAD syndrome: confirmation of linkage to chromosome $4 \mathrm{p}$, evidence 
for locus heterogeneity and a patient with uniparental isodisomy for chromosome 4p. Am J Hum Genet Suppl 1995; 57: 1084.

4 Stratakis C, Taymans SE, Schteingart D, Haddad BR: Segmental uniparental isodisomy (UPD) for 2p16 without clinical symptoms: implications for UPD and other genetic studies of chromosome 2. J Med Genet 2001; 38: 106-109.

5 Tompson SW, Ruiz-Perez VL, Wright MJ, Goodship JA: Ellis-van Creveld syndrome resulting from segmental uniparental disomy of chromosome 4. J Med Genet 2001; 38: e18.

6 Yang XP, Inazu A, Yagi K, Kajinami K, Koizumi J, Mabuchi H: Abetalipoproteinemia caused by maternal isodisomy of chromosome $4 \mathrm{q}$ containing an intron 9 splice acceptor mutation in the microsomal triglyceride transfer protein gene. Arterioscler Thromb Vasc Biol 1999; 19: 1950-1955.

7 Hannula K, Lipsanen-Nyman M, Kontiokari T, Kere J: A narrow segment of maternal uniparental disomy of chromosome 7q31qter in Silver-Russell syndrome delimits a candidate gene region. Am J Hum Genet 2001; 68: 247-253.

8 Martin RA, Sabol DW, Rogan PK: Maternal uniparental disomy of chromosome 14 confined to an interstitial segment (14q2314q24.2). J Med Genet 1999; 36: 633-636.

9 Eggermann T, Mergenthaler S, Eggermann $\mathrm{K}$ et al: Identification of interstitial maternal uniparental disomy (UPD)(14) and complete maternal UPD(20) in a cohort of growth retarded patients. J Med Genet 2001; 38: 86-89.

10 Rio M, Ozilou C, Cormier-Daire V et al: Partial maternal heterodisomy of chromosome 17q25 in a case of severe mental retardation. Hum Genet 2001; 108: 511-515.
11 Avivi L, Korenstein A, Braier-Goldstein O, Goldman B, Ravia Y: Uniparental disomy of sex chromosomes in man. Am J Hum Genet 1992; 51 (Suppl): 33.

12 Lopez-Gutierrez AU, Riba L, Ordonez-Sanchez ML, Ramirez-Jimenez, Cerrillo-Hinojosa M, Tusie-Luna MT: Uniparental disomy for chromosome 6 results in steroid 21-hydroxylase deficiency: evidence of different genetic mechanisms involved in the production of the disease. $J$ Med Genet 1998; 35: 1014-1019.

13 Das S, Lese CM, Song M et al: Partial paternal uniparental disomy of chromosome 6 in an infant with neonatal diabetes, macroglossia, and craniofacial abnormalities. Am J Hum Genet 2000; 67: 1586-1591.

14 Coveler KJ, Yang SP, Sutton R et al: A case of segmental paternal isodisomy of chromosome 14 . Hum Genet 2002; 110: 251-256.

15 Bastepe M, Lane AH, Jüppner H: Paternal uniparental isodisomy of chromosome $20 \mathrm{q}$ - and the resulting changes in GNAS1 methylation - as a plausible cause of pseudohypoparathyroidism. Am J Hum Genet 2001; 68: 1283-1289.

16 Coveler KJ, Sutton VR, Knox-Dubois C, Shaffer LG Comprehensive microsatellite marker analysis contradicts previous report of segmental maternal heterodisomy of chromosome 14. J Med Genet 2003; 40: e26.

17 Christian SL, Fantes JA, Mewborn SK, Huang B, Ledbetter DH Large genomic duplicons map to sites of instability in the Prader-Willi/Angelman syndrome chromosome region (15q11q13). Hum Mol Genet 1999; 8: 1025-1037. 